\title{
Digital Interaction: Where Are We Going?
}

\author{
Tiziana Catarci \\ Sapienza University of Rome \\ Rome, Italy \\ catarci@diag.uniroma1.it \\ Eleonora Bilotta \\ University of Calabria \\ Rende, Italy \\ bilotta@unical.it \\ Antonello Cocco \\ Ministry of Economic Development \\ Rome, Italy \\ antonello.cocco@mise.gov.it

$$
\begin{gathered}
\text { Francesco Di Nocera } \\
\text { Sapienza University of Rom } \\
\text { Rome, Italy }
\end{gathered}
$$ \\ Sapienza University of Rome \\ francesco.dinocera@uniroma1.it \\ Rosa Lanzilotti \\ University of Bari Aldo Moro \\ Bari, Italy \\ rosa.lanzilotti@uniba.it
Pietro S. Pantano
University of Calabria
Rende, Italy
piepa@unical.it

\author{
Massimo Amendola \\ Ministry of Economic Development \\ Rome, Italy \\ massimo.amendola@mise.gov.it \\ Marco Bracalenti \\ University of Perugia \\ Perugia, Italy \\ marco.bracalenti90@gmail.com
University of Bari Aldo Moro
Bari, Italy
maria.costabile@uniba.it \\ Maria Francesca Costabile \\ Stefano Federici \\ University of Perugia \\ Perugia, Italy \\ stefano.federici@unipg.it \\ Andrea Marrella \\ Sapienza University of Rome \\ Rome, Italy \\ marrella@diag.uniroma1.it \\ Isabella Poggi \\ Roma Tre University \\ Rome, Italy \\ isabella.poggi@uniroma3.it
}

\author{
Francesca Bertacchini \\ University of Calabria \\ Rende, Italy \\ frncescabertacchini@live.it \\ Paolo Buono \\ University of Bari Aldo Moro \\ Bari, Italy \\ paolo.buono@uniba.it \\ Giuseppe Desolda \\ University of Bari Aldo Moro \\ Bari, Italy \\ giuseppe.desolda@uniba.it \\ Giancarlo Gaudino \\ Ministry of Economic Development \\ Rome, Italy \\ giancarlo.gaudino@mise.gov.it \\ Maria Laura Mele \\ University of Perugia \\ Perugia, Italy \\ marialaura.mele@gmail.com \\ Laura Tarantino \\ University of L'Aquila \\ L'Aquila, Italy \\ laura.tarantino@univaq.it
}

\begin{abstract}
In the framework of the AVI 2018 Conference, the interuniversity center ECONA has organized a thematic workshop on "Digital Interaction: where are we going?". Six contributions from the ECONA members investigate different perspectives around this thematic.
\end{abstract}

\section{CCS CONCEPTS}

- Human-centered computing $\rightarrow$ Human computer interaction (HCI); Interaction design; Visualization; • Security and privacy $\rightarrow$ Human and societal aspects of security and privacy;

\section{KEYWORDS}

Visual Interfaces, Multimodal Interaction, Accessibility, Usability Evaluation, Participatory Design, User Experience, Human Factors in Cybersecurity

\section{INTRODUCTION}

ECONA is an interuniversity center drawing on the expertise of teaching and researching staff from eight Italian Universities. It is a permanent organization - open to all academic contributions - set up to provide a focus for the study of cognitive processing. ECONA involves a network of academics within a variety of disciplines such as Psychology, Computer Science, Engineering, Maths, 
Physics, Biology, Medicine, Economics and Architecture. Its main goal is to promote multidisciplinary projects in several fields of research including human-computer interaction, psychology of cognitive processes, machine learning, natural language processing, psychophysiology and neuropsychology, modeling of mental processes.

In the framework of the AVI 2018 Conference, ECONA has organized a thematic workshop on "Digital Interaction: where are we going?", accepting contributions from the ECONA members only but open to the public. The various talks aim at providing answers from different perspectives.

A first work surveys many years of research in visual interfaces showing that they are still an indispensable component even in richer interaction environments. Then, another author stresses the importance of being more inclusive by studying interaction modalities and systems for groups of people with special needs, also tailoring standardized tools and techniques to address their needs. A third contribution emphasizes the importance of enriching the interaction with virtual agents (a virtual tourist guide in particular) by reproducing the human guide behaviour in terms of both dialogue and gestures, so offering to the visitors not only information but also an emotional experience. The distance between humans and machines is also investigated in a study aiming at designing objects whose physical characteristics best fit the intended usage to satisfy the users'goals. The study compares users'shape choices with those automatically selected by ML algorithms highlighting the many overlaps. Finally, the still existing need of concentrating on the users when dealing with everyday software is discussed in two talks. The first one makes a step forward in having a more usable Public Administration software by providing the practitioners with a semi-automatic advanced tool for assessing the usability of web sites and services. In the last talk the underestimated role of the human being in the cybersecurity chain is highlighted together with possible solutions to increase the human awareness of the cyber risks and reduce the human errors without sacrificing the system usability.

\section{VISUAL INTERFACES WILL STILL PLAY A KEY ROLE IN FUTURE INTERFACES}

\section{Authors: Paolo Buono, Maria Francesca Costabile.}

The series of AVI conference was started in 1992 by Tiziana Catarci, Maria Francesca Costabile and Stefano Levialdi, with the objective to meet people interested in any type of visual interfaces. After 26 years, the AVI conference is still going very well, attracting world leaders. The current great variety of ICT technology is determining new possibilities for user interfaces. Thus AVI has broadened the topics it covers, still keeping its main focus on the conception, design, implementation and evaluation of novel visual interfaces. AVI success is a further indication that visual interfaces are still able to create valuable user experiences.

The ongoing advances in sensor and display technologies, CPUs, GPUs, and wireless networks are a continuous source of innovation with novel devices ranging from very large displays to small wearables such as smart watches or augmented reality glasses. All these new devices push researchers to envision new interaction possibilities. The proliferation of sensor technology does not only stimulate humans' sense of vision and hearing, but also sense of touch, smell, and taste, challenging to master these senses in creating novel multisensory interfaces. For instance, a recent project discusses how the use of ultrasound mid-air haptic stimulation allowed users to communicate their emotional status. However, researchers working in Virtual Reality (VR) point out that VR systems are now able to create highly visually convincing experiences, they also perform pretty well with the sense of hearing, while current technology is still not able to stimulate our other senses with the same resolution.

We are now more than three decades after the widespread adoption of visual user interfaces with mouse and keyboard. There are more smartphones in the world than desktop PCs and interaction by touch and multi-touch is very popular. One successful example are large interactive displays and whiteboards that rely on pen or touch input and can now be found in many meeting and class rooms or even in public spaces. Over time, size and resolution of such displays increased and they also became far more affordable and widespread, so that the current focus is on the development of more complex applications that meet users' needs in various situations. With the goal of making interaction more âĂIJnaturalâĂİ, new modalities and interaction languages are studied to improve the interaction with the new systems, possibly not mediated by devices such as mouse and keyboard. Performing gestures (or in general body movements) to allow people to communicate with the system is an example. Indeed, advances in computer vision permit real-time body, hand, and finger tracking, making possible to recognize human motions from a distance. In previous work of one of the authors, set of gestures are presented as a type of visual language, suggesting researchers to capitalize on what visual language research has produced since more than thirty years.

A workshop at AVI 2018 considers that multimodal interaction can offer many potential benefits for data visualization. So far, existing visualization techniques have mostly explored a single input modality such as mouse, touch, pen, speech. We agree that it is worth exploiting the strengths of different interaction modalities when analyzing large amounts of data, but proper visual representations have the great potential of enabling people to quickly grasp the content that such data bring, in order to speed up the decision-making process.

Having analyzed various new types of interaction possibilities, this position paper states that visual interfaces will still play a major role in future user interfaces, even if, in some cases, they might be complemented with other interaction modalities within a multimodal interface.

\section{DIGITAL INTERACTION: WHAT IS THE QUESTION? A POSITION ABSTRACT}

\section{Author: Laura Tarantino.}

Speaking about digital interaction, I would like to provocatively turn the proposed question "where are we going" into "where do we want to go", which I find appropriate, giving both the maturity of the discipline and the specific context, i.e., a conference in a field multidisciplinary in its very nature and regarding users as the central aspect of the scientific discourse. Studies focused on successful technological innovation (e.g., $[7,18]$ ) underline the necessity of 
putting emphasis on three legs of human-centered product development, namely user experience, marketing, and technology: an idea must be desirable (user's point of view), viable (business point of view), and feasible (technology point of view). I argue that, while it is a company's interest to ensure the balance of the three supporting legs, the scientific community may - or, to better say, should - foster also unbalanced research, e.g., focusing on specific users' groups deserving community attention and high quality research.

This is for example the case of ICT-enhanced treatment for people with Autism Spectrum Disorder, (see, .e.g., [4, 11, 20] for surveys), which may be regarded as paradigmatic for application domains in which advanced ICT solutions are regarded as highly promising but still a bit at their infancy. This may imply the need of downsizing some experiment parameters considered standard otherwise, e.g., in terms of technological maturity of results (in many case still at the stage of proof-of-concepts) and size of users' groups involved in the evaluation (for example, out of the 38 ICT-based studies surveyed in [4], 20 involved a sample with a size below 10 or not even specified and only 3 have been evaluated with more than 30 persons). While these limitations - along with the speciality of the users population - might suggest to someone that preliminary results are not of interest for the scientific community, it is reasonable to ask ourselves whether it is on the contrary exactly the speciality of the users population and its need and rights for mature studies that should make the scientific community favour studies of this kind in the mainstream of HCI research.

Where do we want to go? Which are our objectives and ethical issues as scientists?

\section{THE MULTIMODAL COMMUNICATION OF ART COMMENTATORS. FROM ANALYSIS TO SIMULATION IN VIRTUAL GUIDES}

\section{Author: Isabella Poggi.}

In the context of the National Project CHROME (Cultural Heritage Resources Orienting Multimodal Experiences), the research unit of Roma Tre is collaborating with the Principal Coordinator Franco Cutugno and the research Unit of Napoli to the construction of Virtual Touristic Guides. The Virtual Guide to be implemented, Maya, is supposed to guide human tourists in a virtual tour of the Chartreuses in Campania, Italy, namely those of S.Martino in Naples, S.Giacomo at Capri, and the Chartreuse of Padula.

While the architect partners of Naples work at a 3D reconstruction of the three Chartreueses through drone grabbing, and engineers build the Virtual guide, the task of Roma Tre is the analysis of the multimodal communicative behavior of human "Art Commentators" (AC): tourist guides, art history experts and other professionals working to illustrate Cultural Heritage resources. The analysis of ACs' multimodal behavior implies on the one side to find out the recurrent structure of their verbal discourse, on the other to analyse the body communicative behavior concomitant to the single parts or aspects of that structure. On the former side, a corpus of ten videos on youtube is collected in which ten ACs illustrate artworks by ancient or contemporary artists in the same TV format. In each video the discourse of the AC is analysed in terms of its hierarchy of goals and a general script is singled out of the goals typically pursued by Art Commentators in presenting an artwork: the "textual goals" of providing information about the work, the Author, and their cultural-historical milieu are aimed at "emotional goals" of triggering emotions in Users, hence bringing about their cultural or spiritual elevation; "textual goals" are pursued, in their turn, through the "modal goals" of soliciting attention, triggering curiosity and interest, facilitating comprehension through lexical definition, vivid illustration, explanations and belief connections.

On the side of body communicative behavior, an annotation scheme is tuned to describe and classify the literal and indirect meanings conveyed by AC's gestures, facial expressions, posture, and gaze. These are finally connected to the specific nodes of AC's discourse: for each body behavior of AC it is assessed whether it contributes to the textual goals of illustrating the artwork (e.g. a deictic gesture pointing at a part of a painting), to the goal of triggering emotions in the User (e.g. a facial expression of enthusiasm or admiration) or finally to one of reconnecting the delivered information to the User's previous beliefs (e.g., an allusive gaze).

The description and classification of the verbal and bodily communication in all ACs in the corpus allows to distinguish their different styles as commentators, both in terms of their preference for textual, emotional or modal goals, and in terms of their use of recurrent verbal or bodily signals. This is finally aimed at reproducing such different styles on the Virtual Guide.

\section{WHAT IS THE FINEST FORM FOR REALIZING THIS PURPOSE? AESTHETICAL-FUNCTIONAL VALUES AS FITNESS FUNCTION}

\section{Authors: Francesca Bertacchini, Pietro S. Pantano, Eleonora Bilotta.}

Besides allowing robots that interact with humans in social environment [5], or realizing visual analysis based on advanced chaotic algorithms [2, 3], one of the main innovation of recent scientific and technological advancements is digitization and digitalization of physical objects [24], process that strongly influences contemporary industrial production. The possibility to parametrize an object allows the related modification of its function as well, by exploring the parameter space of its geometrical configuration. This option, which many CAD systems actually allow, has opened new unpredictable possibility to generate digital 3D shapes of objects, to be used in smart manufacturing [26]. In this technological scenario, many generative algorithms consent to produce thousands of imitative digital objects that vary slightly. However, the problems raise when we want to select the digital objects that best fit with our purpose in order to satisfy the needs of classes of users, in a design process.

We have implemented some computational systems that produce this wealth of digital shapes of objects. We thought to use these objects for smart manufacturing, allowing the process of 3D printing, for physically create new and interesting items for daily use. What is the best digital object to be chosen, having thousands of digital shapes? Sims, in his famous experiments in Computer Graphics [21], explained a method for procedurally generating 3D virtual creatures, using connected graphs, L-systems, and neural networks to generate both morphologies and control behavior. Then, he used aesthetical and functional fitness functions to choose, among a 
huge amount of evolved creatures, the ones that best fit with his purposes. We used a similar procedure of choice made by Sims, employing an empirical fitness that used both the congruence and the appeal of the digital structure to select among thousands of possible shapes. The results must be congruent with the function that the physical object must carry out in the physical environment.

To achieve this goal, we created an experimental situation in which users chose the digital shape they prefer, according to some specified functions. Results have been collected and categorized. In order to compare these results with an automatic choice made by a computational system able to evaluate visual objects, we trained a machine learning system, with different functions, to analyse the same data we have used for human subjects. Unexpectedly, the categories to which the ML arrived by some means overlap with the considered choices made by humans. The study of shared visual elements between artificial systems and humans leads to new interesting perspectives of artificial intelligence which is increasingly closer to the human ones.

\section{UTASSISTANT: A NEW SEMI-AUTOMATIC USABILITY EVALUATION TOOL FOR ITALIAN PUBLIC ADMINISTRATIONS}

Authors: Stefano Federici, Maria Laura Mele, Rosa Lanzilotti, Giuseppe Desolda, Marco Bracalenti, Giancarlo Gaudino, Antonello Cocco, Massimo Amendola.

Since 2013, the Department of Public Function of the Italian Ministry for Simplification and Public Administration (PA) has been developing two usability evaluation protocols designed both for desktop solutions (eGLU 2.1) [8] and mobile platforms (eGLUMobile) [9].

The current work presents a usability evaluation Web platform called UTAssistant ("Usability Tool Assistant") [10], a semiautomatic digital tool developed to support PA web service practitioners in designing usability assessment tests from the outset through the data analysis process, by following the principles and recommendations of both eGLU 2.1 and eGLU-M. UTAssistant aims to provide Italian PA with an easy-to-use tool for assessing the usability of PA websites and services; there is no need to install any software on evaluators' devices, as required by the existing tools for usability testing (e.g., Morae, https://www.techsmith.com/ morae.html [25]).

The fact that UTAssistant is a Web platform represents an important contribution to PA usability assessment, since remote participation fosters wider adoption of these tools and, consequently, of usability testing techniques. UTAssistant supports evaluators (e.g. Web managers of PA websites) of usability assessment procedures in a step-by-step and semi-automatic way. The procedures in UTAssistant follow the protocols used in eGLU 2.1 and eGLU-M, and the experimental methodology used to evaluate the usability of UTAssistant also follows the GLU principles and recommendations by integrating them with new bio-behavioral methods for assessing user interaction. This methodology combines various methods and techniques borrowed from standard usability evaluation procedures and psychophysiological investigation methods using bio-behavioral measures [6].
The new methodology aims to assess usability tools by combining the following: (i) eye-tracking, facial recognition, and electroencephalography measurements [13]; (ii) standard usability evaluation processes, which are compliant with international usability guidelines [6]; (iii) heuristic usability investigations by UX experts [14-17]; and (iv) remote online usability evaluation with highly representative numbers of end-users recruited through Web-based recruitment platforms. The experimental methodology uses biobehavioral variables that are mostly hidden from users, thus overcoming the most common issues that can occur in traditional assessment methodologies, such as the users' tendency to answer questions in a way that is affected by the presumed expectations of the evaluator, i.e. the social desirability bias. UTAssistant, reengineered according to the user-centered evaluation process and complying with ISO/IEC 25010 [1] + eGLU 3.0, forms the content of the eGLU-box service pack.

\section{UNDERSTANDING HUMAN FACTORS IN CYBERSECURITY}

Authors: Tiziana Catarci, Francesco Di Nocera, Andrea Marrella.

The Cyber-Security field investigates solutions for protecting systems, networks, software and data from unauthorized access or attacks aimed at exploitation [22]. The field is growing in its importance due to the increasing presence of connected devices (such as PCs, smartphones, tablets, etc.). The huge amount of data and events produced by the use of those devices dramatically affect the reliability of systems and of exchanged information.

Cyber-security is evolving quickly, and several technological solutions for the design of safer systems are currently being developed. However, technology advances alone can just mitigate the possibility of security breaches, but cannot solve completely all the challenges faced in cybersecurity. To date, the problem is that research in this field tends to neglect that the weakest link in the cybersecurity chain is the human being and her/his limited awareness of the multitude of risks deriving from the interaction with any (modern) technological environment [19].

It is not a coincidence that most of the current attacks are targeting uninformed or misinformed people [12]. The right use of security tools relies on the awareness of their usefulness. Therefore, if users do not understand or are not aware of the security risks, they are more vulnerable to make incorrect behaviours. Moreover, users may be aware of the risk, but may not know which is the correct behaviour. When the user feels overwhelmed by the system demands, s/he could dismiss the system itself [23].

Here we will discuss and tackle this issue by presenting our ongoing research on understanding human factors in cybersecurity. Specifically, we are investigating user-centred foundations and solutions that are specifically tailored to increase cyber-awareness and reduce human errors. Our research is at the intersection of Human-Computer Interaction, Behavior Analysis and Cybersecurity, and is aimed at realizing a "science of usable solutions for cyber-security" that could eventually lead to find the right balance between usability and security. 


\section{REFERENCES}

[1] ISO/IEC 25010:2011. 2011. Systems and Software Engineering - Systems and Software Quality Requirements and Evaluation (Square) - System and Software Quality Models. (2011). Retrieved April 24, 2018 from https://www.iso.org/ standard/35733.html

[2] Marjan Abdechiri, Karim Faez, Hamidreza Amindavar, and Eleonora Bilotta. 2017. The chaotic dynamics of high-dimensional systems. Nonlinear Dynamics 87, 4 (2017).

[3] Marjan Abdechiri, Karim Faez, Hamidreza Amindavar, and Eleonora Bilotta. 2017 Chaotic Target Representation for Robust Object Tracking. Image Commun. 54, C (2017), 23-35

[4] Nuria Aresti-Bartolome and Begonya Garcia-Zapirain. 2014. Technologies as support tools for persons with autistic spectrum disorder: a systematic review. International journal of environmental research and public health 11, 8 (2014).

[5] Francesca Bertacchini, Eleonora Bilotta, and Pietro Pantano. 2017. Shopping with a robotic companion. Computers in Human Behavior 77 (2017).

[6] Simone Borsci, Masaaki Kurosu, Stefano Federici, and Maria Laura Mele. 2013 Computer Systems Experiences of Users with and Without Disabilities: An Evaluation Guide for Professionals (1st ed.). CRC Press, Inc.

[7] Tim Brown. 2009. Change by Design: How Design Thinking Transforms Organizations and Inspires Innovation. Technical Report. Harper Business.

[8] Dipartimento della Funzione Pubblica. 2015. Il Protocollo Eglu 2.1: Come Realizzare Test Di Usabilitá Semplificati Per I Siti Web E I Servizi Online Delle Pa. (2015). Retrieved April 24, 2018 from http://www.funzionepubblica.gov.it/sites/ funzionepubblica.gov.it/files/Protocollo_eGLU_2_1_19082015_DEF_2.pdf

[9] Dipartimento della Funzione Pubblica. 2015. Il Protocollo Eglu-M: Come Realizzare Test Di Usabilitá Semplificati Per I Siti Web E I Servizi Online Delle Pa. (2015). Retrieved April 24, 2018 from http://www.funzionepubblica.gov.it/sites/ funzionepubblica.gov.it/files/Protocollo_eGLU_2_1_19082015_DEF_2.pdf

[10] Giuseppe Desolda, Giancarlo Gaudino, Rosa Lanzilotti, Stefano Federici, and Antonello Cocco. 2017. UTAssistant: A Web Platform Supporting Usability Testing in Italian Public Administrations. In Proceedings of the Doctoral Consortium, Posters and Demos at CHItaly 2017 co-located with 12th Biannual Conference of the Italian SIGCHI Chapter (CHItaly 2017), Cagliari, Italy, September 18-20, 2017.

[11] Ouriel Grynszpan, Patrice L Weiss, Fernando Perez-Diaz, and Eynat Gal. 2014. Innovative technology-based interventions for autism spectrum disorders: a meta-analysis. Autism 18, 4 (2014).
[12] Lee Hadlington. 2017. Human factors in cybersecurity; examining the link between Internet addiction, impulsivity, attitudes towards cybersecurity, and risky cybersecurity behaviours. Heliyon 3, 7 (2017).

[13] Maria Laura Mele and Stefano Federici. 2012. A psychotechnological review on eye-tracking systems: towards user experience. Disability and Rehabilitation: Assistive Technology 7, 4 (2012).

[14] Rolf Molich and Jakob Nielsen. 1990. Improving a Human-computer Dialogue. Commun. ACM 33, 3 (1990).

[15] Jakob Nielsen. 1994. Enhancing the Explanatory Power of Usability Heuristics. In Proceedings of the SIGCHI Conference on Human Factors in Computing Systems (CHI'94). ACM.

[16] Jakob Nielsen and Robert L. Mack (Eds.). 1994. Usability Inspection Methods. John Wiley \& Sons, Inc.

[17] Jakob Nielsen and Rolf Molich. 1990. Heuristic Evaluation of User Interfaces. In Proceedings of the SIGCHI Conference on Human Factors in Computing Systems (CHI'90). ACM.

[18] Donald A Norman. 1998. The invisible computer: why good products can fail, the personal computer is so complex, and information appliances are the solution. MIT press.

[19] Kent L Norman. 2017. Cyberpsychology: An introduction to human-computer interaction. Cambridge university press.

[20] Sarah Parsons and Sue Cobb. 2011. State-of-the-art of virtual reality technologies for children on the autism spectrum. European fournal of Special Needs Education 26, 3 (2011).

[21] Karl Sims. 1994. Evolving virtual creatures. In Proceedings of the 21st annual conference on Computer graphics and interactive techniques. ACM.

[22] Peter W Singer and Allan Friedman. 2014. Cybersecurity: What everyone needs to know. Oxford University Press.

[23] Jeremiah D Still. 2016. Cybersecurity needs you! Interactions 23, 3 (2016).

[24] Fiona Sussan and Zoltan J Acs. 2017. The digital entrepreneurial ecosystem. Small Business Economics 49, 1 (2017).

[25] Craig Tomlin. 2018. 14 Usability Testing Tools Matrix and Comprehensive Reviews. (2018). Retrieved April 24, 2018 from http://www.usefulusability.com/ 14-usability-testing-tools-matrix-and-comprehensive-reviews/

[26] Pai Zheng, Zhiqian Sang, Ray Y Zhong, Yongkui Liu, Chao Liu, Khamdi Mubarok, Shiqiang Yu, Xun Xu, and others. 2018. Smart manufacturing systems for Industry 4.0: Conceptual framework, scenarios, and future perspectives. Frontiers of Mechanical Engineering (2018). 